Relations industrielles

Industrial Relations

\title{
Chaykowski, Richard P. and Anil Verma, eds. Industrial Relations in Canadian Industries
}

\section{Jean Boivin}

Volume 47, numéro 4, 1992

URI : https://id.erudit.org/iderudit/050817ar

DOI : https://doi.org/10.7202/050817ar

Aller au sommaire du numéro

Éditeur(s)

Département des relations industrielles de l'Université Laval

ISSN

0034-379X (imprimé)

1703-8138 (numérique)

Découvrir la revue

Citer ce compte rendu

Boivin, J. (1992). Compte rendu de [Chaykowski, Richard P. and Anil Verma, eds. Industrial Relations in Canadian Industries]. Relations industrielles /

Industrial Relations, 47(4), 792-795. https://doi.org/10.7202/050817ar

Tous droits réservés (C) Département des relations industrielles de l'Université Laval, 1992
Ce document est protégé par la loi sur le droit d'auteur. L'utilisation des services d'Érudit (y compris la reproduction) est assujettie à sa politique d'utilisation que vous pouvez consulter en ligne.

https://apropos.erudit.org/fr/usagers/politique-dutilisation/ 
l'arbitrage de griefs. Peu d'auteurs se sont penchés sur les particularismes de ce régime qui, en quelque sorte, influence directement ou à tout le moins indirectement le quotidien de tous les Québécois. De ce fait, le texte revêt une importance particulière. De plus, il soulève de façon claire et précise, les contradictions du système tel le double rôle de l'État qui est à la fois législateur et employeur. Finalement, l'auteur souhaite une réforme qui permettrait un réel retour aux sources, "c'est-à-dire aux établissements et institutions, aux syndicats locaux et aux gestionnaires mais nullement par voie de délégation condescendante des centrales syndicales et du Conseil du trésor ". II plaide pour un «changement réel d'une praxis, délaissant ainsi quelque stéréotypes révolus».

L'oeuvre dans son ensemble constitue un outil de travail indispensable pour tous ceux, praticiens, professeurs et étudiants, qui s'intéressent à titre de profanes ou de spécialistes aux rapports collectifs du travail. Tous y trouveront leur compte. De plus, et c'est peut-être le plus important à mes yeux, le professeur Morin sait soulever les interrogations et les remises en question qui, par les mutations qu'elles opèrent, suscitent chez le lecteur une réflexion lui permettant de cheminer. Cette démarche me semble beaucoup plus utile que celle de certains auteurs qui prétendent répondre à toutes les questions du lecteur.

Jean-Yves BriérE

Montréal

Industrial Relations in Canadian Industries, by Richard P. Chaykowski and Anil Verma, eds, Toronto, Dryden (Holt, Rinehart and Winston of Canada), 1992, 491 p., ISBN 0-03-922877-0

L'objectif de ce volume est de tenter de cerner les changements survenus dans les relations industrielies au Canada au cours des dix dernières années afin de déterminer si les pratiques en vigueur continueront de s'apparenter à ce que les auteurs appellent le "modèle traditionnel» qui a prédominé de la deuxième Grande Guerre jusqu'au début des années 1980 ou si, au contraire, on peut déceler dans la panoplie de pratiques nouvelles qui se sont développées récemment des indices permettant de conclure à l'apparition d'un nouveau modèle de relations industrielles au Canada. Ce livre fait donc écho à une analyse similaire effectuée il y a quelques années aux États-Unis et qui avait donné lieu à la publication du "best seller» The Transformation of American Industrial Relations, par Kochan, Katz et McKersie (New York, Basic Books, 1986). La préface du volume est d'ailleurs écrite par Thomas Kochan.

Comme dans l'étude portant sur les États-Unis, les deux co-éditeurs s'inspirent de l'approche dite «des choix stratégiques» tout en mettant à contribution l'approche systémique traditionnelle. Cependant, contrairement à The Transformation of American Industrial Relations où les trois auteurs avaient procédé à une étude globale portant sur l'ensemble de l'économie américaine (qui s'appuyait par contre sur les résultats des très nombreuses recherches empiriques auxquelles ils avaient déjà participé), Verma et Chaykowski ont, pour 
leur part, réalisé une dizaine d'analyses sectorielles en tirant profit de la contribution de plusieurs autres chercheurs. Les secteurs analysés sont les suivants: automobile (Kumar et Meltz); acier (Verma et Warrian); mines (Chaykowski); construction (Rose); vêtement (Grant); textile (Thomason, Zwerling et Chandra); secteur public (Thompson et Ponak); éducation (Downie); aéronautique (Fisher et Kondra); et téléphone (Verma et Weiler). On peut déplorer en passant l'absence de certains secteurs comme celui du papier qui constitue pourtant la principale industrie canadienne en termes d'emplois. Cette omission ainsi que celle des secteurs de la foresterie, des produits pétroliers et de la santé est d'ailleurs reconnue par les auteurs qui l'expliquent par le manque de données disponibles.

Les caractéristiques essentielles des différentes industries sont présentées dans un chapitre synthèse qui utilise un modèle permettant d'apprécier les différents stages d'évolution des relations industrielles des secteurs étudiés. II s'agit là d'ailleurs d'une contribution originale qui vient enrichir l'approche stratégique développée par Kochan, Katz et McKersie.

La qualité des textes a aussi bénéficié d'une conférence organisée par les co-éditeurs en avril 1991 où les auteurs des différentes monographies ont soumis les résultats de leurs travaux à l'appréciation et à la critique de praticiens et d'académiciens du milieu des relations industrielles avant que les manuscrits ne soient finalisés.

Chacun des auteurs avait à apprécier l'impact de la globalisation des marchés et de l'environnement de plus en plus concurrentiel dans lequel opèrent les entreprises sur les pratiques de relations industrielles et de gestion des ressources humaines. On a cherché à savoir si les entreprises canadiennes avaient adopté des stratégies visant à réduire la présence et l'importance syndicale comme ce fut le cas aux États-Unis, si elles avaient introduit des programmes de participation et d'intéressement des employés ou encore si elles s'étaient engagées dans des négociations conduisant à des alliances stratégiques avec les syndicats pour faire face aux nouveaux défis causés par l'environnement. Les co-éditeurs ont repris les résultats de ces analyses dans le chapitre final pour essayer d'identifier les facteurs responsables de la relative stabilité des pratiques de relations industrielles dans certaines industries (téléphone) comparativement aux bouleversements profonds survenus dans d'autres secteurs (construction dans l'ouest du Canada, vêtement). Dans la plupart des cas étudiés cependant, il semble que les parties soient rendues à un point critique où elles devront faire des choix décisifs afin de déterminer si elles désirent continuer de fonctionner selon lapproche traditionnelle de relations adversariales ou plutôt s'engager sur la voie de discussions pouvant conduire à des alliances stratégiques.

Aux États-Unis, la faiblesse de la densité syndicale a rendu ce dernier choix plutôt exceptionnel, les entreprises préférant diminuer l'influence syndicale par tous les moyens possibles. Au Canada où la densité syndicale est plus élevée, les syndicats ont manifesté beaucoup de méfiance vis-à-vis toutes discussions pouvant conduire à des alliances stratégiques. Dans la mesure où la performance économique des entreprises n'a pas encore atteint le point de crise, les syndicats ont pu maintenir avec une certaine efficacité leur approche traditionnelle en relations industrielles comme en témoigne l'expérience de 
l'industrie automobile, même si dans ce dernier cas, on retrouve au niveau local beaucoup plus d'ouverture aux nouvelles pratiques de gestion que dans les discours officiels de la direction nationale du syndicat. Cependant, avec la libéralisation de plus en plus marquée des échanges commerciaux (que l'on pense au nouveau traité de libre-échange Canada-États-Unis-Mexique), plusieurs entreprises dans des secteurs comme l'acier, le textile et laéronautique sont sur le point d'atteindre ce point de crise. Les choix que les syndicats auront à faire seront sans doute très douloureux: s'ils maintiennent leurs pratiques traditionnelles, ils courent le risque de voir les entreprises opter pour des stratégies d'évitement de la syndicalisation comme ce fut le cas aux États-Unis, même si, à court terme, ils peuvent maintenir leurs droits chèrement acquis dans le passé. D'autre part, s'ils acceptent de s'engager sur la voie conduisant à des alliances stratégiques, ils n'ont aucune garantie que cette attitude permettra de sauver les emplois à long terme, même si cette démarche peut donner des résultats positifs à court terme.

L'immense avantage de ce livre (par rapport à The Transformation of American Industrial Relations) c'est qu'il permet de situer chacune des industries étudiées sur un continuum dont le point de départ est constitué de ce que les éditeurs appellent «le système traditionnel» et le point d'arrivée "les alliances stratégiques". Entre ces deux pôles extrêmes, on retrouve les cinq points intermédiaires suivants: "les pressions pour le changement"; "la diminution de la performance économique de l'entreprise"; "les concessions mineures non accompagnées de changements d'attitude»; «le point de crise" où l'on retrouve des changements d'attitude qui entraîneront des modifications importantes à la négociation collective; et enfin, ales négociations stratégiques" qui conduiront éventuellement aux alliances stratégiques. Les auteurs prennent cependant bien soin d'ajouter qu'il existe deux autres scénarios beaucoup moins intéressants pour les syndicats mais qui sont tout aussi plausibles comme alternatives à la négociation et aux alliances stratégiques, à savoir: les fermetures ou le désinvestissement systématique dans les établissements syndiqués d'une part; ou encore l'érosion graduelle de la représentation syndicale et même la disparition du syndicat comme cela s'est déroulé aux États-Unis.

Les auteurs ont très bien identifié les pressions responsables des changements apportées dans les pratiques de relations industrielles et de gestion des ressources humaines. Ils ont également bien décrit les réponses des acteurs et le livre contient un résumé succinct des différentes innovations apportées dans les différentes industries (que ce soit en termes de flexibilité du travail, de sécurité d'emploi et du revenu, de participation des employés ou de formes de rémunération). L'inclusion de deux chapitres sur le secteur public est très judicieuse car elle permet de constater que les pressions de l'environnement ont également atteint ce secteur. 
Industrial Relations in Canadian Industry est donc une contribution majeure à la connaissance du système contemporain de relations industrielles canadien et il sera apprécié tant comme lecture de base pour les cours portant sur la négociation collective que comme lecture complémentaire pour les cours portant sur la théorie des relations industrielles.

Jean BoIvin

Université Laval

Industrial Relations in Canadian Industry, by Richard $P$. Chaykowsky and Anil Verma eds, New York, The Dryden Press, 1992, 491 p., ISBN 0-03-922877-0

Industrial Relations in Canadian Industry, edited by Richard P. Chaykowski and Anil Verma, analyzes the transformation (or nontransformation) of industrial relations in ten Canadian sectors, using Kochan, Katz, and McKersie's strategicchoice model. The book thus supplies a hitherto missing link between industrial relations theory and practice at the industry level, which industrial relations academics and practioners alike will appreciate. Furthermore, the application of a common framework of analysis facilitates meaningful comparisons among sectors in the extent and nature of their transformation toward new systems of industrial relations. A sectoral focus also highlights the importance of industrylevel public policies, actor relationships, and external pressures in shaping common industrial relations outcomes. The book thus accurately portrays the pluralism within the national system of industrial relations during the economic upheavals of the last decade.

The book is subdivided into twelve chapters. The first chapter describes both the theoretical tools for analyzing the different industries and the social, economic, and industrial relations developments at the national level which serve as the background to the industry studies. Chapters two through eleven specify the pressures for change in the auto, steel, mining, construction, clothing, textile, public service, education, airline, and telecommunications sectors, the coping responses of strategic decision-makers, and their implications for industrial relations. Chapter twelve categories the experiences of these ten sectors as differing stages, within a modified strategic-choice framework, in the transformation away from a traditional adversarial system of industrial relations toward several potential new alternatives.

In chapter one, Richard P. Chaykowski and Anil Verma expound the virtues of Kochan, Katz, and McKersie's strategic-choice approach as a method of analyzing industrial relations in the ten sectors studied. They argue that industrial relations developments are best understood within the wider context of strategic decision-making in marketing, production, and finance rather than in the narrower context of collective bargaining alone. For this reason, the authors describe the national context of industrial relations in terms of, for example, trade, productivity, labour costs, and public policies, while leaving the authors of subsequent chapters to describe industry-specific characteristics relevant to industrial relations. 